ARTICLE

\title{
Restoration of rhythmicity in diffusively coupled dynamical networks
}

\author{
Wei Zou ${ }^{1,2,3}$, D.V. Senthilkumar ${ }^{3,4}$, Raphael Nagao ${ }^{5}$, István Z. Kiss ${ }^{5}$, Yang Tang ${ }^{3,6}$, Aneta Koseska ${ }^{7,8}$, \\ Jinqiao Duan ${ }^{1,2,9}$ \& Jürgen Kurths $3,10,11,12$
}

Oscillatory behaviour is essential for proper functioning of various physical and biological processes. However, diffusive coupling is capable of suppressing intrinsic oscillations due to the manifestation of the phenomena of amplitude and oscillation deaths. Here we present a scheme to revoke these quenching states in diffusively coupled dynamical networks, and demonstrate the approach in experiments with an oscillatory chemical reaction. By introducing a simple feedback factor in the diffusive coupling, we show that the stable (in)homogeneous steady states can be effectively destabilized to restore dynamic behaviours of coupled systems. Even a feeble deviation from the normal diffusive coupling drastically shrinks the death regions in the parameter space. The generality of our method is corroborated in diverse non-linear systems of diffusively coupled paradigmatic models with various death scenarios. Our study provides a general framework to strengthen the robustness of dynamic activity in diffusively coupled dynamical networks.

\footnotetext{
${ }^{1}$ School of Mathematics and Statistics, Huazhong University of Science and Technology, Wuhan 430074, China. ${ }^{2}$ Center for Mathematical Sciences, Huazhong University of Science and Technology, Wuhan 430074, China. ${ }^{3}$ Potsdam Institute for Climate Impact Research, Telegraphenberg, D-14415 Potsdam, Germany. ${ }^{4}$ Centre for Nonlinear Science and Engineering, School of Electrical and Electronics Engineering, SASTRA University, Thanjavur 613401, India. ${ }^{5}$ Department of Chemistry, Saint Louis University, 3501 Laclede Avenue, St Louis, Missouri 63103, USA. ${ }^{6}$ The Key Laboratory of Advanced Control and Optimization for Chemical Processes, Ministry of Education, East China University of Science and Technology, Shanghai 200237, China. ${ }^{7}$ Department of Systemic Cell Biology, Max Planck Institute of Molecular Physiology, Dortmund D-44227, Germany. ${ }^{8}$ Research Centre for Computer Science and Information Technologies, Macedonian Academy of Sciences and Arts, Skopje, Macedonia. ${ }^{9}$ Department of Applied Mathematics, Illinois Institute of Technology, Chicago, Illinois 60616, USA. ${ }^{10}$ Institute of Physics, Humboldt University Berlin, D-12489 Berlin, Germany. ${ }^{11}$ Institute for Complex Systems and Mathematical Biology, University of Aberdeen, Aberdeen AB24 3FX, UK. ${ }^{12}$ Department of Control Theory, Nizhny Novgorod State University, Gagarin Avenue 23, 606950 Nizhny Novgorod, Russia. Correspondence and requests for materials should be addressed to W.Z. (email: zouwei2010@mail.hust.edu.cn) or to I.Z.K. (email: izkiss@slu.edu).
} 
$\mathrm{M}$ odelling coupled oscillators offer an alternative and effective approach aimed at understanding a plethora of intriguing self-organizing phenomena in diverse fields of non-linear sciences ranging from physics, chemistry, biology and neuroscience to engineering ${ }^{1-4}$. The phenomenon of oscillation quenching (oscillation suppression) is a fascinating emerging behaviour, whereby individual oscillatory systems cease to oscillate when coupled, which results into the emergence of stationarity of the whole-coupled system ${ }^{5,6}$. One of the early observations of oscillation quenching dates back to the 19th century by Lord Rayleigh, who found that two organ pipes of the same pitch standing side by side mutually suppressed their vibration ${ }^{2}$. Later, this phenomenon was observed in chemical reactions ${ }^{7,8}$, lasers ${ }^{9}$, electronic circuits ${ }^{10}$, neural oscillators ${ }^{11}$ and so on. Models based on coupled non-linear oscillators have been extensively used to understand the intricacies of oscillation quenching for more than two decades ${ }^{5,6}$. Very recently, two structurally distinct oscillation quenching processes have been clearly distinguished as: (i) amplitude death (AD) and (ii) oscillation death (OD) $)^{6,12}$. Generally, $\mathrm{AD}$ refers to stabilization of an already existing homogeneous steady state (HSS) to which the coupled units are entrained. In contrast, OD is manifested as a novel inhomogeneous steady state (IHSS), which occurs due to symmetry breaking of the coupled systems, and the distinct units then populate different branches of the same IHSS ${ }^{6,12}$.

The phenomena of $\mathrm{AD}$ and $\mathrm{OD}$ can be responsible for a loss of intrinsic dynamics, which may lead to a large degree of degradation in the dynamic performance of physical systems. For example, the onset of $\mathrm{AD}$ and $\mathrm{OD}$ could seriously weaken and even completely destroy the transmission of electricity in a power grid, where the energy flows along transmission lines arise from periodic oscillations of the rotational phase of generators at the frequency of $50 \mathrm{~Hz}(60 \mathrm{~Hz})^{13-15}$. On the other hand, sustained oscillations represent one of the most striking and ubiquitous manifestations of dynamic evolution in distinct real-world systems $s^{16,17}$, such as spiking neural networks, cardiac and respiratory systems, and El Niño/Southern Oscillation in Earth's ocean and atmosphere and so on. Moreover, rhythmic behaviours play an essential function at all levels of physiological processes, which make it possible to organize and control the activity of populations of cells ${ }^{18}$. For instance, it has been proposed that highly synchronized theta frequency oscillations serve as a temporal organizer for cortex in the hippocampus ${ }^{19}$. These examples furnish the robustness of dynamic activity as a main prerequisite for proper functioning of multitude natural and man-made networks. Thus, oscillation quenching in such real systems is destructive and fatal to robustness of rhythmic behaviours, which is undesirable and should be circumvented.

For a couple of decades, the major research focus was centred at identifying various scenarios that facilitate the emergence of $\mathrm{AD}$ and $\mathrm{OD}$ in coupled oscillatory systems ${ }^{20-34}$. Only in the recent past, a significant attention has been devoted to explore possibilities of restoring rhythmic activity after the dynamic activity of the coupled networks irreversibly fails, as a means to understand sustained oscillatory mechanisms of natural systems ${ }^{35-39}$. However, a general technique to efficiently overcome the phenomenon of oscillation quenching in coupled oscillatory systems remains as an open challenge as highlighted in a recent review ${ }^{5}$.

In this paper, we propose a rather simple and efficient approach for revoking oscillation suppression: a limiting factor in the diffusive coupling effectively recovers rhythmic behaviour of coupled networks, whose dynamic activity has been destroyed by the diffusive interaction of the elements. We demonstrate that this simple limiting factor can destabilize the stable HSS and IHSS to wipe out the onset of both $\mathrm{AD}$ and $\mathrm{OD}$ in diffusively coupled paradigmatic non-linear oscillators. Astonishingly, even a minute deviation from the normal diffusive coupling can reduce drastically the stable regimes of $\mathrm{AD}$ and $\mathrm{OD}$ in the parameter space. Both the scheme and the phenomenon are clearly verified in experiments using electrochemical oscillators: the stability of the HSS of current generating chemical reactions emanating from dissolution of nickel wires is revoked by a simple adjustment of the nature of the interactions. The proposed method is highly efficient and quite robust in regaining sustained oscillations in networks of diffusively coupled non-linear oscillators, where the dynamic activity has been damaged and lost as a consequence of the diffusive interaction. Our study leads to a significant improvement of our understanding of the role of diffusive coupling in controlling oscillatory activity of coupled complex non-linear systems.

\section{Results}

Two coupled Stuart-Landau oscillators. In the following, we will demonstrate our approach using the paradigmatic model of coupled Stuart-Landau oscillators, describing dynamics near a supercritical Hopf bifurcation (further examples of oscillatory systems are discussed in the Supplementary Notes 1-5). Systems of coupled Stuart-Landau oscillators have served as an ideal model for exploring the mechanisms of $\mathrm{AD}$ by analytical means for more than a couple of decades ${ }^{20-28}$. So far, numerous scenarios such as frequency mismatch ${ }^{20,21}$, time-delayed coupling $22-26$, and conjugate and dynamic couplings ${ }^{27,28}$ and so on have been reported to induce AD in coupled Stuart-Landau oscillators. On the other hand, a burst of recent research activity has revealed that OD can even occur in coupled identical StuartLandau oscillators when the coupling destroys the rotational symmetry of the system ${ }^{12,31-34}$. Here we will establish that the onset of both $\mathrm{AD}$ and $\mathrm{OD}$ under all the above-mentioned scenarios can be effectively revoked to retain self-sustained oscillations by a very simple coupling scheme.

Let us begin with two coupled Stuart-Landau oscillators ${ }^{1}$

$$
\begin{aligned}
\dot{Z}_{j}(t)= & \left(1+i w_{j}-\left|Z_{j}(t)\right|^{2}\right) Z_{j}(t) \\
& +K\left[Z_{k}(t-\tau)-\alpha Z_{j}(t)\right],
\end{aligned}
$$

where $j, k=1,2(j \neq k), Z_{j}=x_{j}+i y_{j}$ and $w_{j}$ are the complex amplitude and the natural frequency of the $j$ th oscillator, respectively, $K$ is the coupling strength, and $\tau$ is the propagation delay. In contrast to the studies on the traditional diffusive interaction, here we introduce a new factor $\alpha$ in the coupling. The new ingredient offers the opportunity to better characterize the diffusion process at the interface of a wide range of natural systems. In addition, the new feedback factor $\alpha$ acts as a control parameter: $\alpha=1$ corresponds to a normal diffusive coupling, whereas $\alpha=0$ refers to a direct coupling scheme. Thus, the new diffusive coupling may naturally occur in more realistic circumstances as a bridge-linking direct coupling and normal diffusive interaction. We will mainly probe its influence on the collective dynamics of coupled non-linear systems, and reveal that an extremely small detuning of $\alpha$ from the normal diffusion has a significant impact on the oscillatory activity of the system.

For zero-coupling strength $(K=0)$, the $j$ th Stuart-Landau oscillator exhibits a stable limit-cycle oscillation $Z_{j}=e^{i w_{j} t}$ and has an unstable focus at $Z_{j}=0$. The occurrence of $\mathrm{AD}$ in the coupled system (Equation 1) for $\alpha=1$ has been well studied in ref. 20 for $\tau=0$ and in ref. 22 for $\tau>0$. In this respect, Aronson et al. ${ }^{20}$ asserted that $\mathrm{AD}$ occurs for sufficiently disparate frequencies, whereas Reddy et al. ${ }^{22}$ showed that AD occurs even for coupled identical oscillators if $\tau>0$. We next show that the coupling with 
$\alpha<1$ overrides the quenching effects by revoking the stability of $\mathrm{AD}$ to regain oscillations in the respective $\mathrm{AD}$ parameter space.

When the coupled system (Equation 1) experiences $\mathrm{AD}$, the HSS at the origin is stabilized. The condition for the onset of AD can be determined from a linear stability analysis around $Z_{1}=Z_{2}=0$. Assuming that the linear perturbations vary as $e^{\lambda t}$, the characteristic equation can be written as

$$
\left(1+i w_{1}-\alpha K-\lambda\right)\left(1+i w_{2}-\alpha K-\lambda\right)-K^{2} e^{-2 \lambda \tau}=0 .
$$

Thus, for $\tau=0$, the stability condition of $\mathrm{AD}$ is: $1 / \alpha<K<\gamma(\alpha)$, where $\gamma(\alpha)=\left(1+\Delta^{2} / 4\right) / 2 \quad$ if $\quad \alpha=1$ and $\gamma(\alpha)=\left(-\alpha+\sqrt{1+\left(1-\alpha^{2}\right) \Delta^{2} / 4}\right) /\left(1-\alpha^{2}\right)$ if $\alpha<1$, where $\Delta=$ $\left|w_{1}-w_{2}\right|$. We find that the spread of the stable HSS (AD) in the $(K, \alpha)$ space narrows down on decreasing $\alpha$ for $\Delta>2$ (Fig. 1a for $\Delta=5)$ and eventually vanishes if $\alpha<\alpha_{\min }$. The value of $\alpha_{\min }$ can be analytically deduced as $\alpha_{\min }=2 / \Delta$, and exactly agrees with the simulation results in Fig. 1a. The exact fit between the simulation $\alpha_{\min }$ (black squares) and the analytical $\alpha_{\min }$ (red line) is depicted in Fig. $1 \mathrm{~b}$ as a function of $\alpha$ and $\Delta$. Thus, it is clear that the feedback factor $\alpha$ plays a crucial role in determining the stability of HSS, which restores oscillations in stable HSS (AD) regimes by revoking its stability for $\alpha<\alpha_{\min }$ in coupled nonidentical oscillators.

The characteristic equation (2) can be further simplified as

$$
\lambda=1+i w-\alpha K \pm K e^{\lambda \tau},
$$

for $w_{1}=w_{2}=w$, whose roots can be analytically resolved as

$$
\lambda=\frac{1}{\tau} W\left( \pm \tau K e^{-(1+i w-\alpha K) \tau}\right)+1+i w-\alpha K,
$$
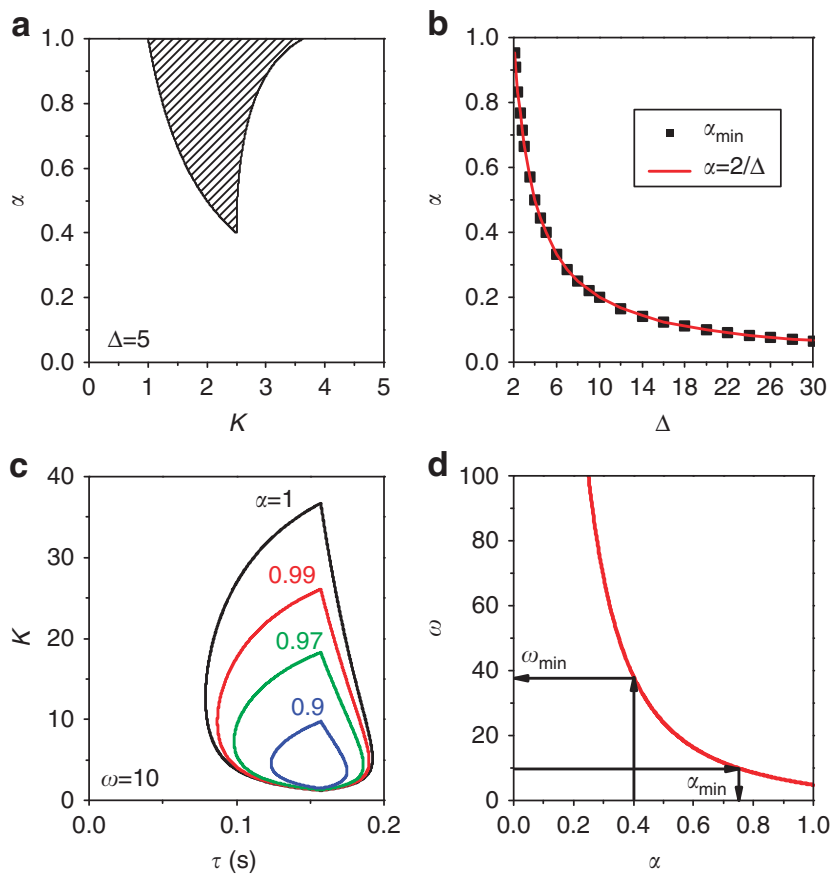

Figure 1 | AD with frequency mismatch and discrete time delay. (a) The stable AD interval versus $\alpha$ with frequency mismatch $\Delta=\left|w_{1}-w_{2}\right|=5$ and $\tau=0$. AD is impossible for any coupling strength $K$ with $\alpha<\alpha_{\min }=0.4$.

(b) The critical $\alpha_{\min }$ versus frequency mismatch $\Delta$ for $\tau=0$. (c) AD islands in the parameter $(\tau, K)$ space for different values of $\alpha$ with $w_{1}=w_{2}=w=10$. (d) The minimal value $\alpha_{\min }$ versus $w$. If $\alpha<\alpha_{\min } A D$ is completely revoked for all $\tau$ and $K$. where $W$ is the Lambert function defined as the inverse map of $G(\Lambda)=\Lambda e^{\Lambda}$ (ref. 40). Moreover, the boundaries of stable HSS (AD islands) on the $(\tau, K)$ plane for $w_{1}=w_{2}=w$ can be explicitly derived as

$$
\begin{gathered}
\tau_{1}=\frac{\cos ^{-1}\left(\frac{\alpha K-1}{K}\right)}{w-\sqrt{K^{2}-(1-\alpha K)^{2}}}, \\
\tau_{2}=\frac{\pi-\cos ^{-1}\left(\frac{\alpha K-1}{K}\right)}{w+\sqrt{K^{2}-(1-\alpha K)^{2}}} .
\end{gathered}
$$

AD islands in the $(\tau, K)$ plane for $\alpha=1,0.99,0.97$ and 0.9 are illustrated in Fig. 1c for $w=10$. Surprisingly, we observe that the $\mathrm{AD}$ island shrinks greatly as $\alpha$ is slightly decreased, and completely disappears if $\alpha<\alpha_{\min } \approx 0.748$. Now, the relation between $\alpha_{\min }$ and $w$ can be deduced as

$$
w=\min \left\{\frac{\pi \sqrt{K^{2}-(1-\alpha K)^{2}}}{\pi-2 \cos ^{-1}\left(\frac{\alpha K-1}{K}\right)}: K>\frac{1}{\alpha}\right\},
$$

which is depicted in Fig. 1d using the red line. This line demarcates the unstable HSS on its left side for any combinations of $(\tau, K)$ and stable HSS on its right side for a certain set of $(\tau, K)$. A minimal $\alpha_{\min }$ necessary for revoking the stability of HSS for a given $w$ on the $(\tau, K)$ plane and vice versa can also be determined from the above relation. Hence, it is strongly confirmed that the feedback factor $\alpha$ successfully induces oscillations in stable HSS (AD) regimes of delay-coupled identical oscillators for $\alpha<\alpha_{\min }$.

Atay ${ }^{25}$ reported that distributed delays favour a stabilization of HSS in a larger parameter space compared with discrete delays. In what follows, we will explore the impact of the feedback factor $\alpha$ on the two coupled Stuart-Landau oscillators with distributed delays

$$
\begin{aligned}
\dot{Z}_{j}(t) & =\left(1+i w_{j}-\left|Z_{j}(t)\right|^{2}\right) Z_{j}(t) \\
& +K\left[\int_{0}^{\infty} f\left(\tau^{\prime}\right) Z_{k}\left(t-\tau^{\prime}\right) d \tau^{\prime}-\alpha Z_{j}(t)\right],
\end{aligned}
$$

where $f$ represents a distributed delay kernel. If $f$ is the delta function $f\left(\tau^{\prime}\right)=\delta\left(\tau^{\prime}-\tau\right)$, one can recover the coupled system (Equation 1). Here we will concentrate on $w_{1}=w_{2}=w$, and a uniformly distributed delay kernel $f\left(\tau^{\prime}\right)=1 /(2 \beta)$ if $\left|\tau^{\prime}-\tau\right|<\beta$ and zero elsewhere as in ref. 25. The kernel $f$ approaches $\delta\left(\tau^{\prime}-\tau\right)$ as $\beta \rightarrow 0$. For $\alpha=1$, Atay $^{25}$ showed that the $\mathrm{AD}$ region becomes unbounded along $\tau$ when $\beta>0.008$ for $w=30$ as illustrated in Fig. $2 \mathrm{a}$ with $\beta=0.02$. To our surprise, on a very small decrease of $\alpha$ from 1, we find that the spread of the AD regime shrinks sharply as shown in Fig. $2 b, c$ for $\alpha=0.99$ and 0.98 , respectively. Moreover, the stable AD region breaks into three disjoint and bounded islands for $\alpha=0.97$ (Fig. 2d). Decreasing $\alpha$ further wipes off the $\mathrm{AD}$ region completely from the whole parameter space $\left(\alpha<\alpha_{\min }=0.424\right)$, thereby restoring stable oscillations by switching the stability of stable HSS (AD). Hence, the feedback factor $\alpha$ circumvents $\mathrm{AD}$ in coupled oscillators even for time delays distributed over an interval, thereby corroborating the robust nature of $\alpha$ in invoking oscillations by revoking the stability of $\mathrm{AD}$.

Conjugate and dynamic couplings are two forms of novel and distinct interactions facilitating AD in coupled identical oscillators without any propagation delays ${ }^{27,28}$. Could the presence of the feedback factor $\alpha$ be capable of eradicating the onset of AD in the coupled systems with both coupling configurations? To clarify it, we thus consider the following systems of Stuart-Landau 
a

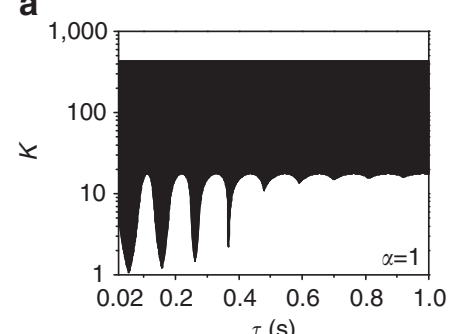

C

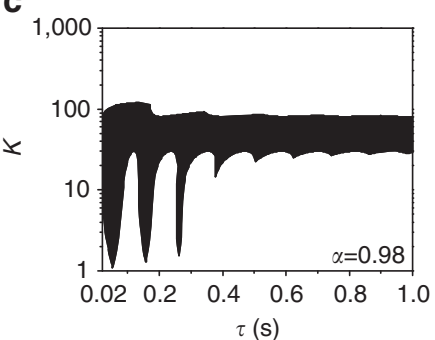

b

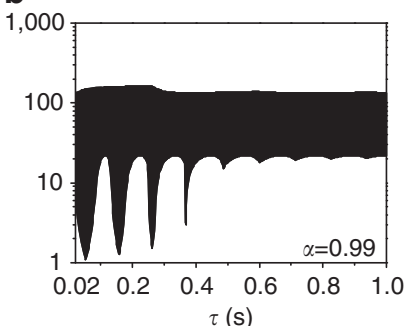

d

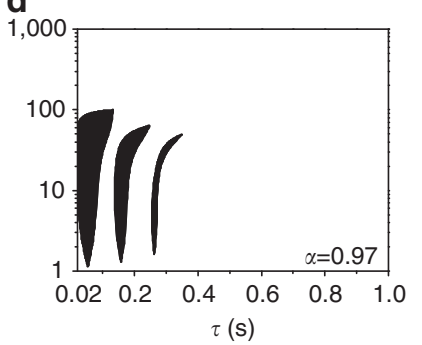

Figure 2 | AD with distributed time delays. (a-d) The stability region of AD with time delays uniformly distributed over $\tau \pm 0.02$ for $\alpha=1,0.99$, 0.98 and 0.97 , respectively. The stable AD region breaks into three disconnected and bounded islands for $\alpha=0.97$. A feeble decrease in the value of the limiting factor $\alpha$ from unity drastically shrinks the stable regions of $A D$ in the parameter space, which implies that dynamic activity is efficiently restored. AD is impossible for any values of $\tau$ and $K$ if $\alpha<\alpha_{\min }=0.424$. The intrinsic frequencies are fixed as $w_{1}=w_{2}=w=30$.

oscillators with conjugate coupling

$$
\begin{aligned}
& \dot{x}_{j}=p_{j} x_{j}-w y_{j}+K\left(y_{k}-\alpha x_{j}\right), \\
& \dot{y}_{j}=w x_{j}+p_{j} y_{j}+K\left(x_{k}-\alpha y_{j}\right),
\end{aligned}
$$

where $p_{j}=1-\left|Z_{j}\right|^{2}=1-x_{j}^{2}-y_{j}^{2}, j, k=1,2 \quad(j \neq k)$, and with dynamic coupling

$$
\begin{aligned}
& \dot{Z}_{j}=\left(1+i w-\left|Z_{j}\right|^{2}\right) Z_{j}+K\left(\mu_{j}-\alpha Z_{j}\right), \\
& \dot{\mu}_{j}=-\mu_{j}+Z_{k} .
\end{aligned}
$$

The underlying mechanisms of $\mathrm{AD}$ induced by conjugate and dynamic couplings are completely different compared to that of the previous cases. In conjugate coupling, the individual systems are coupled via dissimilar variables ${ }^{27}$, whereas additional variables are involved to describe the dynamic coupling ${ }^{28}$. For both coupling scenarios, $\mathrm{AD}$ can be identified by performing a linear stability analysis around the origin of the coupled systems (Equations 8 and 9). The corresponding characteristic equations can be obtained as

$$
(1-\alpha K-\lambda)^{2}+w^{2}-K^{2}=0
$$

and

$$
\lambda^{2}-(i w-\alpha K) \lambda-1-i w-(1-\alpha) K=0,
$$

respectively. For conjugate coupling (Equation 8 ), $\mathrm{AD}$ occurs in the interval $1 / \alpha<K<\gamma(\alpha)$, where $\gamma(\alpha)=\left(1+w^{2}\right) / 2$ if $\alpha=1$ and $\gamma(\alpha)=\left(-\alpha+\sqrt{1+\left(1-\alpha^{2}\right) w^{2}}\right) /\left(1-\alpha^{2}\right)$ if $\alpha<1$. Stable HSS (AD) is achieved only for $w>1$, but monotonically decreases with $\alpha$, which is wiped off for $\alpha<\alpha_{\min }=1 / w$. Figure 3a depicts the spread of the AD interval in the $(K, \alpha)$ space for $w=5$, which strictly decreases with decreasing $\alpha$ and vanishes at $\alpha_{\min }=0.2$. The critical values of $\alpha_{\min }$ below which the coupled oscillators retain their oscillations for all $K>0$ are shown in Fig. $3 \mathrm{~b}$ as a function of $w$. The theoretical prediction (red line) is in good
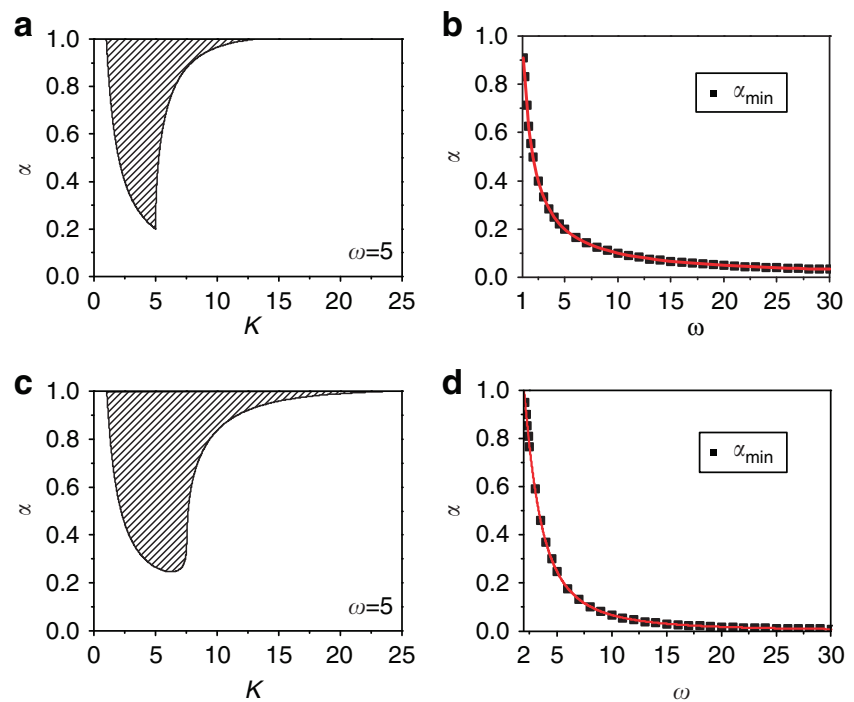

Figure 3 | AD with conjugate and dynamic couplings. (a,c) The stable AD interval $K$ versus $\alpha$ for conjugate and dynamic couplings, respectively. $w=5$ is fixed. $(\mathbf{b}, \mathbf{d})$ The minimal $\alpha_{\text {min }}$ versus $w$ for conjugate and dynamic couplings, respectively. The black squares denote numerical results, and the red line represents theoretical predictions. The scheme for restoration of rhythmicity is valid for coupled systems with distinctly different interactions.

agreement with the numerical results (black squares) (Fig. 3b). For dynamic coupling Equation 9, the stable interval of HSS (AD) is $\left(w^{2}-w \sqrt{w^{2}-4}\right) / 2<K<\left(w^{2}+w \sqrt{w^{2}-4}\right) / 2$ when $\alpha=1$, which requires $w>2$ for the coupled system to experience $\mathrm{AD}$. On decreasing $\alpha$, the stable interval of HSS monotonically reduces (Fig. 3c), which ceases to exist if $\alpha<\alpha_{\min }=\left[w^{2}\left(w^{2}+9\right)-w \sqrt{\left(w^{2}-3\right)^{3}}\right] /\left[2\left(w^{2}+1\right)^{2}\right]$. This theoretical relation (red line) agrees very well with the simulation results (black squares) as depicted in Fig. 3d. Thus, the effect of the feedback factor $\alpha$ in revoking $\mathrm{AD}$ to restore the natural harmonics of the coupled systems can be generally applied to distinct coupling configurations for which a stable AD is possible.

Next, we explore the capability of the feedback factor $\alpha$ in effectively retrieving rhythmic oscillations in systems where OD has been observed. Consider the following system of two StuartLandau oscillators via the $x$ component ${ }^{12}$,

$$
\begin{aligned}
& \dot{x}_{j}=p_{j} x_{j}-w y_{j}+K\left(x_{k}-\alpha x_{j}\right), \\
& \dot{y}_{j}=w x_{j}+p_{j} y_{j},
\end{aligned}
$$

where $p_{j}=1-\left|Z_{j}\right|^{2}=1-x_{j}^{2}-y_{j}^{2}, j, k=1,2(j \neq k)$. Here the onedimensional diffusive coupling involves only the real parts. This coupling form breaks the rotational symmetry of the coupled system, which is a necessary condition for the emergence of OD. Besides the HSS at the origin $Z_{1}=Z_{2}=0$ in the system (Equation 12), the system also displays an IHSS $\left(x^{\star}, y^{*},-x^{*},-y^{*}\right)$ that appears at $K=\left(1+w^{2}\right) /(1+\alpha)$ via a pitchfork bifurcation, with $x^{*}=p \sqrt{(1-p) /\left(w^{2}+p^{2}\right)}$ and $y^{*}=-w \sqrt{(1-p) /\left(w^{2}+p^{2}\right)} \quad$ with $p=(1+\alpha) K / 2-$ $\sqrt{(1+\alpha)^{2} K^{2}-4 w^{2}} / 2$. For the normal diffusive coupling, $\alpha=1$, it was reported that the origin is unstable for all $K>0$, but the IHSS (OD) is stabilized for $K>w^{2}+1 / 4$ (refs 12,31); this is demonstrated by the bifurcation diagram plotted in Fig. 4a with $w=10$. The bold red lines denote the stable branches of the IHSS and the thin lines refer to the unstable ones. We further 
a

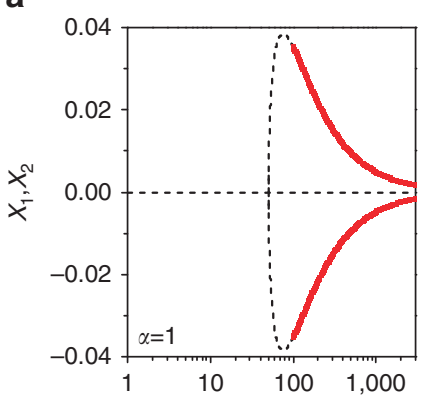

d

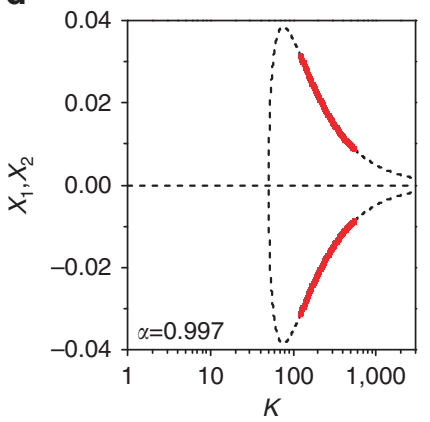

b

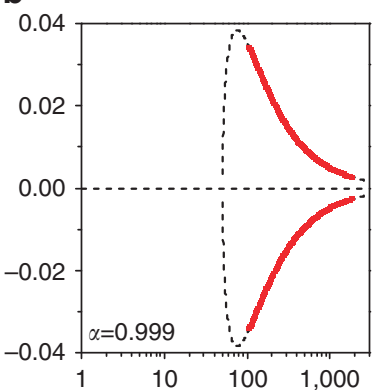

e

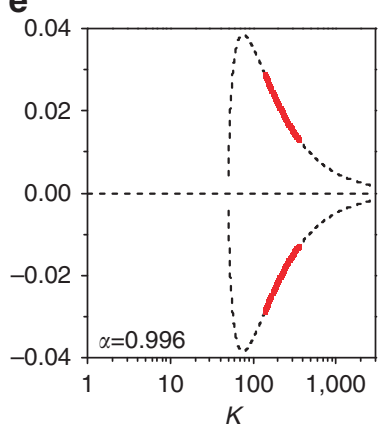

c

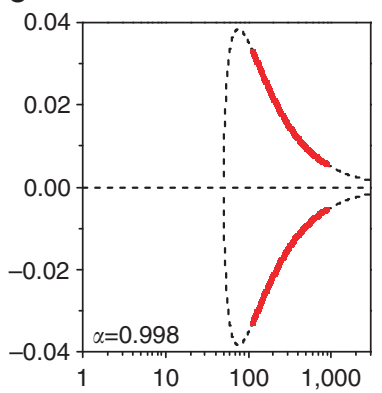

f

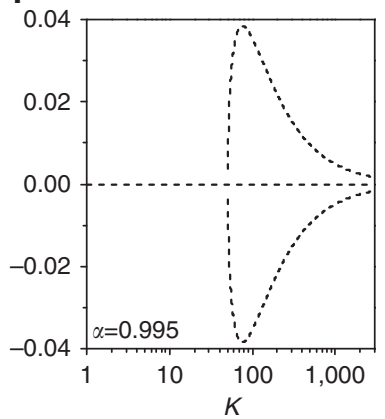

Figure 4 | OD with one-dimensional diffusive coupling via the $x$ component. (a-f) The bifurcation diagrams of the steady-state solutions for $\alpha=1,0.999$, 0.998, 0.997, 0.996 and 0.995, respectively. The bold red lines represent stable IHSS (OD), and the thin black lines denote unstable steady states. Even an infinitesimal change of $\alpha$ from unity drastically shrinks the stable OD interval, which shows the high efficiency of the method in restoring rhythmic activity. The frequency is used as $w=10$.

depict the bifurcation diagrams of the steady-state solutions for $\alpha=0.999,0.998,0.997,0.996$ and 0.995 in Fig. 4b-e, respectively. Astonishingly, we observe that even a very tiny detuning in $\alpha$ from 1 shrinks the coupling intervals of the stable IHSS (OD) (Fig. 4b-e). Moreover, no stable IHSS can be found for any coupling strength when $\alpha<\alpha_{\min } \approx 0.995$ (Fig. 4f), which attributes that $\mathrm{OD}$ is successfully revoked and thus oscillations are provoked in the OD regimes. The effect of the limiting factor $\alpha$ in restoration of rhythmic activity in this case is even more pronounced, since infinitesimal changes of $\alpha$ from unity lead to a sharp shrinkage of the stable IHSS regime, demonstrating the high efficiency of the proposed method in restoring oscillatory activity.

By studying diverse systems of two coupled Stuart-Landau oscillators, it has been demonstrated that incorporating the limiting feedback factor $\alpha$ in the normal diffusive coupling is a rather simple and high-efficient approach in revoking not only $\mathrm{AD}$ but also $\mathrm{OD}$ under various distinct death scenarios. Even a very minute deviation of $\alpha$ from unity drastically shrinks both $\mathrm{AD}$ and OD regions in the parameter space. The new coupler with $\alpha$ strengthens the tolerance to different dynamic deteriorations of coupled non-linear oscillators.

Networked coupled Stuart-Landau oscillators. The role of the diffusive feedback factor $\alpha$ in revoking AD persists for an arbitrary network of coupled Stuart-Landau oscillators. To illustrate it, let us consider a connected network of $N$ Stuart-Landau oscillators with an arbitrary topology

$$
\begin{aligned}
\dot{Z}_{j} & =\left(1+i w_{j}-\left|Z_{j}\right|^{2}\right) Z_{j} \\
& +\frac{K}{d_{j}} \sum_{k=1}^{N} g_{j k}\left[\int_{0}^{\infty} f\left(\tau^{\prime}\right) Z_{k}\left(t-\tau^{\prime}\right) d \tau^{\prime}-\alpha Z_{j}\right]
\end{aligned}
$$

where $j=1,2, \ldots, N$ and $f$ is the uniformly distributed delayed kernel as in equation 7 . The parameter $g_{j k}$ determines the topology of the network, that is, $g_{j k}=g_{k j}=1$ if $j$ th and $k$ th nodes are linked otherwise $g_{j k}=g_{k j}=0, g_{j j}=0$; and $d_{j}=\sum_{k=1}^{N} g_{j k}$ is the $j$ th node degree. The coupled system (Equation 13) $(\alpha=1)$ was studied by Atay for all-to-all networks ${ }^{25}$, that is, $g_{j k}=1$ for all $j \neq k$. Performing a linear stability analysis around the origin, the characteristic equations determining the stability of the HSS in coupled system (Equation 13) with $w_{j}=w$ are

$$
\lambda=1+i w-\alpha K+K \rho_{j} e^{-\lambda \tau} \frac{\sinh (\lambda \beta)}{\lambda \beta},
$$

where $\rho_{j}$ 's are the eigenvalues of $G=\left(\frac{g_{j k}}{d_{j}}\right)_{N \times N}$ and can be ordered as $1.0=\rho_{1} \geq \rho_{2} \geq \ldots \geq-\frac{1}{N-1} \geq \rho_{N} \geq-1.0$ (ref. 41). The network (Equation 13) experiences $\mathrm{AD}$ when the largest real part of roots of equation (14) is negative for all $\rho_{j}$. In fact, only two bounding eigenvalues $\rho_{1}=1$ and $-1 /(N-1) \geq \rho_{N} \geq-1$ contribute to the stability regions of $\mathrm{AD}$. The degree of the spread of the stable HSS is determined by $\rho_{N}$; the spread of AD is larger for larger values of $\rho_{N}$. The stable HSS regime (AD) experienced by the coupled system (Equation 13) for $\alpha=1$ as $\rho_{N} \rightarrow 0$ is plotted in Fig. 5a for uniformly distributed delays with $\beta=0.07$ and $w=10$. The $\mathrm{AD}$ regime is connected and unbounded along $\tau$. We are indeed surprised to observe that the $\mathrm{AD}$ regime becomes disconnected even for a negligible decrease in $\alpha$; see Fig. $5 b$ for $\alpha=0.999$. Decreasing $\alpha$ further, the number of $\mathrm{AD}$ islands decreases as shown in Fig. 5c,d for $\alpha=0.998$ and 0.99, respectively. The $\mathrm{AD}$ region completely disappears in the parameter space for $\alpha<\alpha_{\min } \approx 0.189$, thereby revoking the stability of HSS (AD) in recovering oscillations in an arbitrary network with distributed time delays.

Experiments with chemical oscillations. The efficiency of the new coupling approach in revoking deaths to restore oscillations 
a

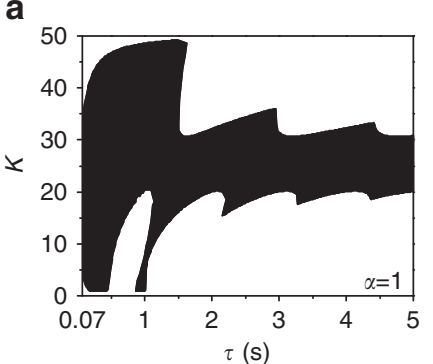

C

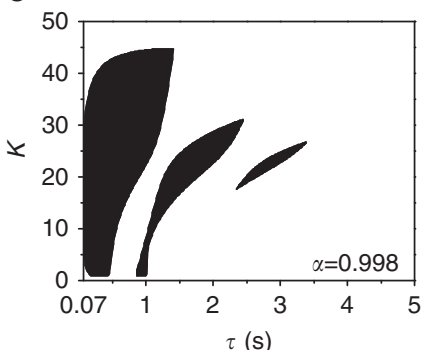

b

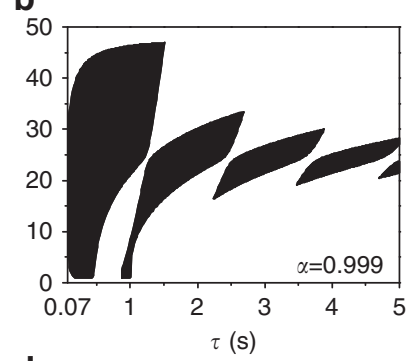

d

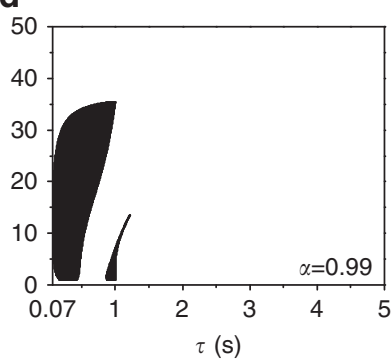

Figure 5 | $A D$ in an arbitrary network with distributed time delays.

(a-d) The upper limiting stability region of $A D$ as $\rho_{N} \rightarrow 0$ with time delays uniformly distributed over $\tau \pm 0.07$ for different values of $\alpha$. AD regime becomes disconnected even for a tiny decrease of $\alpha$ from 1 to 0.999 . Only three AD islands survive for $\alpha=0.998$. Decreasing $\alpha$ further, the number of $A D$ islands decreases. The AD region completely disappears in the parameter space if $\alpha<\alpha_{\min }=0.189$. The intrinsic frequency is fixed as $w=10$.

has been experimentally verified in an oscillatory chemical reaction system with electrochemical dissolution of nickel in sulfuric acidic media. Figure 6a depicts a schematic view of the experimental setup. The reactions take place on the surface of two nickel wires with diameter of $0.69 \mathrm{~mm}$, spaced by $2.0 \mathrm{~mm}$ and embedded in epoxy resin. When an individual resistance of $2.50 \mathrm{k} \Omega$ are attached to each wire, and the potential $V_{0}$ of each wire are set with a bipotentiostat (Bank Instruments) to $1.150 \mathrm{~V}$ versus $\mathrm{Hg} / \mathrm{Hg}_{2} \mathrm{SO}_{4} / \mathrm{sat}$. $\mathrm{K}_{2} \mathrm{SO}_{4}$ reference electrode, oscillatory current can be recorded with the built-in ammeters of the bipotentiostat between the wires and the counter electrode ( $1.6 \mathrm{~mm}$ diameter Pt coated Ti wire). This potential value is just above (by $25 \mathrm{mV}$ ) a Hopf bifurcation. The current oscillations of the two electrodes $\left(I_{1}(t)\right.$ and $\left.I_{2}(t)\right)$, shown in Fig. 6b, occur due to the non-linear kinetics of nickel dissolution in the presence of nickel-oxide films that provide hidden negative differential resistance characteristics to the electrochemical process ${ }^{42,43}$.

The interactions between the chemical reactions on the two metal surfaces are introduced by polarizing the two electrodes (indexed as $j=1$ and 2) according to the following equations:

$$
V_{j}(t)=V_{0}+K\left[<\bar{I}(t-\tau)>-\alpha \bar{I}_{j}(t)\right]
$$

where $\bar{I}_{j}(t)$ are the offset corrected currents of the electrodes $\left(\bar{I}_{j}(t)=I_{j}(t)-o\right), o$ is the average current overtime determined before the experiments, and $\langle\bar{I}(t)\rangle=\left(\bar{I}_{1}(t)+\bar{I}_{2}(t)\right) / 2$ is the mean current of oscillations at time $t$. The potential perturbations, with values of $\alpha$ and $K$ previously chosen with a Labview Real Time software, are applied at a rate of $1,000 \mathrm{~Hz}$, which is much faster than the typical timescale of the oscillations $(2.5 \mathrm{~s})$.

Without control, the chemical reactions on the two electrodes take place in an oscillatory manner with slightly different periods $(2.504 \mathrm{~s}$ and $2.524 \mathrm{~s})$, as shown in Fig. 6b. Because of the lack of coupling, the oscillations exhibit phase drift behaviour (Supplementary Fig. 1) ${ }^{43}$. When the control is turned on with

a
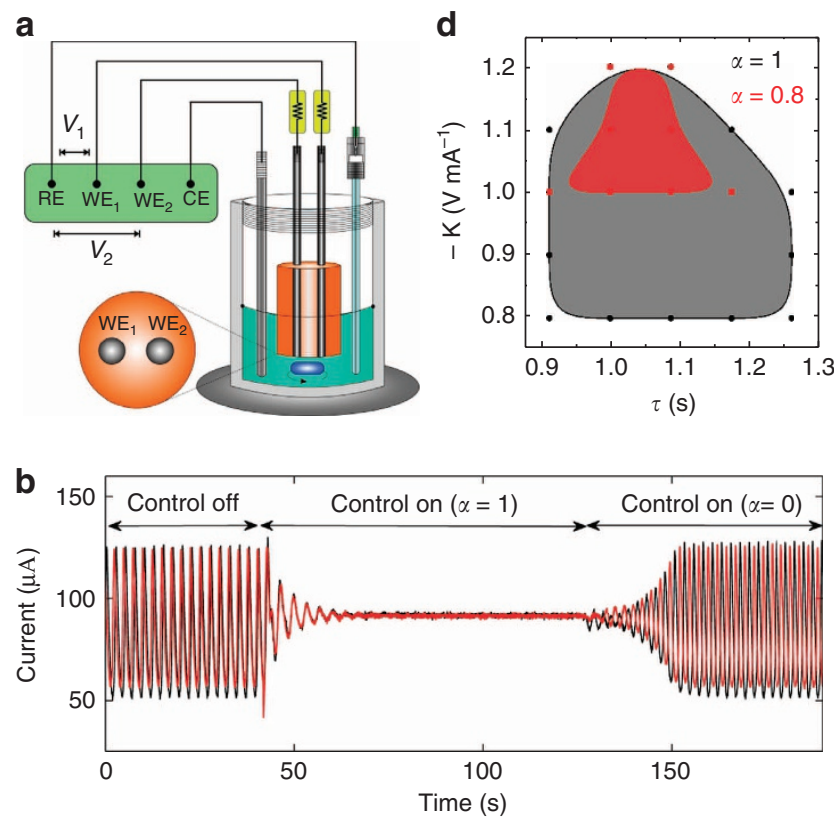

C

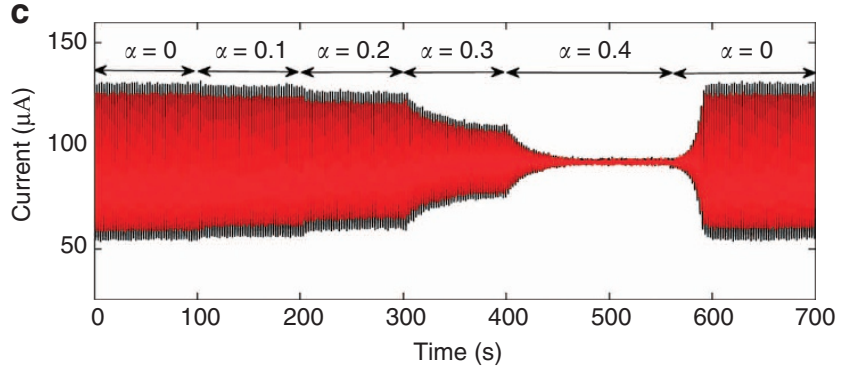

Figure 6 | Experiments with coupled electrochemical reactions.

(a) Schematic of the experimental setup containing the reference (RE), counter (CE) and two Ni working electrodes (WE) in an electrochemical cell with $50 \mathrm{ml}^{3} \mathrm{~mol} \mathrm{~L}^{-1}$ sulfuric acid at a temperature of $10^{\circ} \mathrm{C}$.

(b) Establishing $A D$ with $\alpha=1$ and regaining oscillations with $\alpha=0$. Without control, the oscillations phase drift $(t<40.13 \mathrm{~s}, K=0)$. With direct feedback control AD is observed $\left(40.13 \mathrm{~s}<t<127.1 \mathrm{~s}, K=-1.0 \mathrm{VmA}^{-1}\right.$, $\tau=1.09 \mathrm{~s}$ and $\alpha=1$ ). With $\alpha=0$, the oscillations are anti-phase synchronized. ( $t>127.1 \mathrm{~s}, K=-1.0 \mathrm{VmA}^{-1}, \tau=1.09 \mathrm{~s}$ and $\alpha=0$ ).

(c) Current time series with several values of $\alpha=0,0.1,0.2,0.3$ and 0.4 . (d) AD domains for $\alpha=1$ and 0.8 in the parameter space for $K$ versus $\tau$.

The set of discrete data points are approximated by a cubic spline interpolation, which indicates the border between $A D$ and oscillatory states.

$\alpha=1$, the amplitude quickly decreases, and the system establishes a stable HSS: the delayed interaction thus causes the phenomenon of AD. The oscillations are reborn in an anti-phase configuration when $\alpha$ is adjusted to 0 for $t>127.1 \mathrm{~s}$. Corroborating with this scenario, the gradual increase of $\alpha$, shown in Fig. 6c, clearly indicates a promoting effect on $\mathrm{AD}$. For $\alpha<0.4$, the oscillatory behaviour can be reinstated. Figure $6 \mathrm{~d}$ depicts the AD domains for two values of $\alpha=1$ and 0.8 in $K$ versus $\tau$ diagram. As predicted by our theory, a small decrease of $\alpha$ markedly suppresses the $\mathrm{AD}$ regions.

The experimental realization of our method with chemical oscillations was successful, despite the fact that while the StuartLandau system is a simple, weakly non-linear, two variable model, the chemical system represents an ensemble of complex physical and chemical processes with many variables and uncertainties. The oscillations in the chemical reactions generally originate from a Hopf bifurcation, which can be well represented by the 
Stuart-Landau equations. Therefore, it is expected that the phenomenon of retrieving oscillations with our scheme will work for a large number of other chemical and biological systems, whose oscillatory behaviour arises as a result of a Hopf bifurcation ${ }^{44}$.

\section{Discussion}

We have proposed a rather simple and efficient coupling scheme to revoke both $\mathrm{AD}$ and $\mathrm{OD}$ to retrieve rhythmic behaviours in diffusively coupled dynamical networks of non-linear oscillators. By introducing a limiting feedback factor $\alpha$ in the diffusive coupling, we have revealed that this approach is generically robust in revoking $\mathrm{AD}$ in coupled Stuart-Landau oscillators under distinct scenarios such as frequency mismatch, delayed coupling with both discrete and distributed time delays, and conjugate and dynamic couplings. We have shown that the OD phenomenon can also be revoked in coupled Stuart-Landau oscillators. Intriguingly, a minimal decrease of $\alpha$ from unity drastically reduces the size of the stable regions of both $\mathrm{AD}$ and $\mathrm{OD}$ in the parameter space. The effect of a minute deviation of $\alpha$ from unity manifests in switching the stability of the stable HSS and IHSS. Furthermore, we have experimentally confirmed the efficient role of the diffusive control factor $\alpha$ in restoring oscillations by destabilizing $\mathrm{AD}$ in a delay-coupled chemical reaction system with $\mathrm{Ni}$ electrodissolution.

The surprising effect of a minute deviation of the parameter $\alpha$ from unity could be interpreted in the following intuitive way. In the death scenarios, the normal diffusive coupling $(\alpha=1)$ brings a strong additional dissipation into the coupled oscillatory systems to suppress the oscillations. In our proposed diffusive coupling, the value of $\alpha$ describes the relation between the incoming and the outgoing flows. Specifically, the level of incoming flow is measured by $K$, whereas the outgoing one by $K \alpha$. Clearly, the rate of the incoming flow is greater than that of the outgoing one if $0<\alpha<1$, which leads to asymmetric flows in the whole-coupled systems once $\alpha$ deviates from unity. The surplus incoming flow supplies an internal energy source to compensate the dissipation of coupled systems, which serves as a driving force to induce oscillatory activity.

It is to be noted that we have corroborated our results by employing the paradigmatic Stuart-Landau oscillator, which represents a normal form describing dynamics near a supercritical Hopf bifurcation. Our findings are expected to hold true for a broad class of coupled non-linear systems near a Hopf bifurcation. In fact, we have confirmed that dynamic activity can be recovered effectively in many other diffusively coupled non-linear oscillators experiencing $\mathrm{AD}$ or $\mathrm{OD}$, such as Brusselators (Supplementary Note 1), chaotic Lorenz oscillators (Supplementary Note 2), Pikovsky-Rabinovich circuit models (Supplementary Note 3), synthetic genetic relaxation oscillators and membrane models (Supplementary Note 4). The generality of our method has also been successfully validated in diffusively coupled dynamical networks experiencing distinctly different deteriorations of dynamic activity such as partial deaths ${ }^{45}$ (Supplementary Note 5) and aging transition ${ }^{46,47}$ (Supplementary Note 6). Thus, the new ingredient $\alpha$ in the coupling serves as a very general framework to strengthen the robustness of dynamic activity in diffusively coupled networks.

Our proposed coupling scheme has important consequences in particular in biology. Recent studies of synthetic biology have made significant progress in the construction of populations of intracellular oscillators that mimic naturally occurring clocks as a means to gain insight about the functionality of biological networks ${ }^{48,49}$. In this regard, it would be beneficial to develop sensing compartments, which are non-difference $(\alpha=0)$ for maintaining the oscillations. Assuming that the intrinsic dynamics of a cell is oscillatory, this means that it is important to develop isolated reference states inside the cells where the periodic variations of the cell life cycle are less affected. In neural networks, two major types of coupling forms are: gap junctions and chemical synapses ${ }^{50}$. Gap junctions are based on the potential difference between the cells $(\alpha=1)$, while chemical synapses are through substances generated by action potential and thus do not directly depend on the potential difference $(\alpha=0)$. Our prediction indicates that chemical synapses play a crucial role in maintaining the oscillations, and by changing the ratio of gap junction and electrical coupling could be a versatile way to tune the extent of oscillations in the networks. Our study also has important implications for the design of coupling schemes for synchronization engineering, that is, for tuning the phase relationship between oscillatory units. Feedback-based algorithms can be applied directly or through difference schemes $^{51}$. In general, to avoid AD, direct feedback methods could provide wider parameter ranges at strong feedback gains where synchronization structures are to be attained without amplitude suppression ${ }^{52}$

A population of coupled non-linear oscillators are deemed to serve as an efficient tool to enhance our understanding of collective dynamics spontaneously emerging in real-life systems. The coupling in an ensemble of non-linear oscillators could be designed in different ways. In particular, diffusive coupling is a natural and fundamental type of interaction, which has attracted great interests in different context in a wide range of disciplines including physics, chemistry and biology for many decades. Communication between cells and most of biological process occur through diffusion of ions. In chemical reactions, mixing of different reactants generally involves diffusive interactions. Therefore, in this work, we have considered the diffusive coupling as a starting point of our study in revealing restoration of oscillations of coupled networks. By introducing a new parameter modifying the nature of the normal diffusive coupling, we have elaborated that even a very tiny deviation from the traditional diffusive coupling causes a drastic influence on the dynamic activity of coupled dynamical systems. Our results illuminate the role of diffusive coupling in the generation of sustained rhythmic activity in real-world systems. It was reported that the phenomena of $\mathrm{AD}$ and $\mathrm{OD}$ are also possible for coupled nonlinear systems with non-diffusive couplings ${ }^{53-57}$. How to revoke $\mathrm{AD}$ and $\mathrm{OD}$ in non-diffusively coupled systems constitutes our next task to be studied.

Finally, our proposed coupling scheme can be easily realized not only in different model studies but also for diverse experimental setups. We firmly speculate that our approach is feasible in other distinct experiments, where $\mathrm{AD}$ or $\mathrm{OD}$ has been reported ${ }^{58-61}$. The non-trivial influence of the new diffusive coupling scheme on the qualitative properties of dynamical systems indicates a new path for future studies of other collective behaviours in diffusively coupled non-linear oscillators such as chimera states $^{62,63}$, explosive synchronization ${ }^{64,65}$ and glass states $^{66-68}$. The framework of our study sheds significantly new insights on the diffusive coupling in manipulating oscillatory dynamics of coupled complex non-linear systems, which will have a strong impact and invoke wide interests in the field of complex systems science as well as in various applications from biology via engineering to social sciences.

\section{Methods}

Numerical simulations. All numerical integrations of coupled dynamical equations are performed by employing a standard explicit fourth-order Runge-Kutta method with integration steps $h=0.01$ and 0.001 , respectively. There are no qualitative differences between two different steps. Random initial conditions can 
be adopted for $\mathrm{AD}$ in coupled Stuart-Landau oscillators, which means that $\mathrm{AD}$ is not only linearly but also globally stable in the phase space. In contrast, as OD always coexists with stable limit-cycle motions, initial conditions near the IHSS are used for OD, or many different random initial conditions are tried until reaching $\mathrm{OD}$.

Steady-state solutions for AD and OD. The onset of AD in coupled StuartLandau oscillators implies that the unstable origin is stabilized by the coupling. Thus the steady-state solution for AD in coupled Stuart-Landau oscillators is HSS at $Z_{j}=0$. In contrast, OD manifests due to the appearance of stable IHSS. In general, for OD in coupled non-linear oscillators, the Newton-Raphson algorithm ${ }^{69}$ is used to locate the IHSS solution. In coupled Stuart-Landau oscillators in equation (12), the IHSS solution for OD is analytically derived, which is also confirmed by the Newton-Raphson algorithm.

Linear stability analysis. The occurrences of $\mathrm{AD}$ and $\mathrm{OD}$ are verified from a standard linear stability analysis after obtaining steady-state solutions of $\mathrm{AD}$ and OD in coupled Stuart-Landau oscillators. AD (OD) is stable, if and only if all the real parts of resulted characteristic eigenvalue equations are negative. Once a time delay is involved, the corresponding characteristic eigenvalue equations are transcendental, which have infinitely many (both complex and real) roots with negative real parts, but only a finite number of roots with positive real parts attributed to instability. Generally, the characteristic roots of transcendental characteristic equations cannot be solved analytically. We numerically compute the largest real part of characteristic roots with the pseudospectral differentiation techniques ${ }^{70}$. For one special case, the characteristic roots can be expressed explicitly using the Lambert function $W$ as indicated in equation (4).

AD boundaries. The AD boundaries in equation (5) are analytically derived. The analysis is based on the fact that as tuning $\tau$ or $K$, the stability of HSS in coupled Stuart-Landau oscillators may be switched only if a characteristic eigenvalue root $\lambda$ in equation (3) crosses the imaginary axis. For the critical situation, we have $\lambda=i \Omega$. Inserting into equation (3) and separating into real and imaginary part, the $\mathrm{AD}$ boundary curves can be obtained after some straightforward algebraic manipulations.

The critical value of $\alpha_{\min }$. The values of $\alpha_{\min }$ theoretically predicted in equation (6) are from the intersection condition of $\mathrm{AD}$ boundaries in equation (5). Generally, the threshold value of $\alpha_{\min }$ in all cases is calculated by meticulously decreasing $\alpha$ from 1 until the stable regime of HSS (AD) or IHSS (OD) is extinct in the whole parameter space.

\section{References}

1. Kuramoto, Y. Chemical Oscillations. Waves, and Turbulence (Springer, 1984).

2. Pikovsky, A., Rosenblum, M. \& Kurths, J. Synchronization: A Universal Concept in Nonlinear Sciences (Cambridge Univ. Press, 2001).

3. Strogatz, S. H. From Kuramoto to Crawford: exploring the onset of synchronization in populations of coupled oscillators. Physica. D 143, 1-20 (2000).

4. Acebrón, J. A., Bonilla, L. L., Vicente, C. J. P., Ritort, F. \& Spigler, R. The Kuramoto model: a simple paradigm for synchronization phenomena. Rev. Mod. Phys. 77, 137 (2005).

5. Saxena, G., Prasad, A. \& Ramaswamy, R. Amplitude death: the emergence of stationarity in coupled nonlinear systems. Phys. Rep. 521, 205-228 (2012).

6. Koseska, A., Volkov, E. \& Kurths, J. Oscillation quenching mechanisms: amplitude vs oscillation death. Phys. Rep. 531, 173-199 (2013).

7. Bar-Eli, K. \& Reuveni, S. Stable stationary states of coupled chemical oscillators. experimental evidence. J. Phys. Chem. 89, 1329-1330 (1985).

8. Crowley, M. F. \& Epstein, I. R. Experimental and theoretical studies of a coupled chemical oscillator: phase death, multlstability, and in-phase and outof-phase entrainment. J. Phys. Chem. 93, 2496-2502 (1989).

9. Kuntsevich, B. F. \& Pisarchik, A. N. Synchronization effects in a dualwavelength class-B laser with modulated losses. Phys. Rev. E 64, 046221 (2001).

10. Reddy, D. V. R., Sen, A. \& Johnston, G. L. Experimental evidence of time-delayinduced death in coupled limit-cycle oscillators. Phys. Rev. Lett. 85, 3381-3384 (2000).

11. Ermentrout, G. B. \& Kopell, N. Oscillator death in systems of coupled neural oscillators. SIAM J. Appl. Math. 50, 125-146 (1990).

12. Koseska, A., Volkov, E. \& Kurths, J. Transition from amplitude to oscillation death via Turing bifurcation. Phys. Rev. Lett. 111, 024103 (2013).

13. Döfler, F., Chertkov, M. \& Bullo, F. Synchronization in complex oscillator networks and smart grids. Proc. Natl Acad. Sci. USA 110, 2005-2010 (2013).

14. Motter, A. E., Myers, S. A., Anghel, M. \& Nishikawa, T. Spontaneous synchrony in power-grid networks. Nat. Phys. 9, 191-197 (2013).

15. Menck, P. J., Heitzig, J., Kurths, J. \& Schellnhuber, H. J. How dead ends undermine power grid stability. Nat. Commun. 5, 3969 (2014)

16. Jenkins, A. Self-oscillation. Phys. Rep. 525, 167-222 (2013).
17. Tchumatchenko, T. \& Clopath, C. Oscillations emerging from noise-driven steady state in networks with electrical synapses and subthreshold resonance. Nat. Commun. 5, 5512 (2014).

18. Koukkari, W. L. \& Sothern, R. B. Introducing Biological Rhythms (Springer, 2006).

19. Lisman, J. \& Buzsaki, G. A neural coding scheme formed by the combined function of gamma and theta oscillations. Schizophr. Bull. 34, 974-980 (2008).

20. Aronson, D. G., Ermentrout, G. B. \& Kopell, N. Amplitude response of coupled oscillators. Physica. D 41, 403-449 (1990).

21. Mirollo, R. E. \& Strogatz, S. H. Amplitude death in an array of limit-cycle oscillators. J. Stat. Phys. 60, 245-262 (1990).

22. Reddy, D. V. R., Sen, A. \& Johnston, G. L. Time delay induced death in coupled limit cycle oscillators. Phys. Rev. Lett. 80, 5109-5112 (1998).

23. Reddy, D. V. R., Sen, A. \& Johnston, G. L. Time delay effects on coupled limit cycle oscillators at Hopf bifurcation. Physica. D 129, 13-34 (1999).

24. Strogatz, S. H. Death by delay. Nature 394, 316-317 (1998).

25. Atay, F. M. Distributed delays facilitate amplitude death of coupled oscillators. Phys. Rev. Lett. 91, 094101 (2003).

26. Zou, W. \& Zhan, M. Partial time-delay coupling enlarges death island of coupled oscillators. Phys. Rev. E 80, 065204 (2009).

27. Karnatak, R., Ramaswamy, R. \& Prasad, A. Amplitude death in the absence of time delays in identical coupled oscillators. Phys. Rev. E 76, 035201(R) (2007).

28. Konishi, K. Amplitude death induced by dynamic coupling. Phys. Rev. E 68, 067202 (2003)

29. Prigogine, I. \& Lefever, R. Symmetry breaking instabilities in dissipative systems. J. Chem. Phys. 48, 1695-1700 (1968).

30. Bar-Eli, K. On the stability of coupled chemical oscillators. Physica. D 14, 242-252 (1985).

31. Zakharova, A. et al. Time delay control of symmetry-breaking primary and secondary oscillation death. Europhys. Lett. 104, 50004 (2013).

32. Zakharova, A., Kapeller, M. \& Schöll, E. Chimera death: symmetry breaking in dynamical networks. Phys. Rev. Lett. 112, 154101 (2014).

33. Zou, W., Senthilkumar, D. V., Koseska, A. \& Kurths, J. Generalizing the transition from amplitude to oscillation death in coupled oscillators. Phys. Rev. E 88, 050901 (2013).

34. Zou, W., Senthilkumar, D. V., Duan, J. \& Kurths, J. Emergence of amplitude and oscillation death in identical coupled oscillators. Phys. Rev. E 90, 032906 (2014).

35. Konishi, K. Limitation of time-delay induced amplitude death. Phys. Lett. A 341, 401-409 (2005).

36. Morino, K., Tanaka, G. \& Aihara, K. Efficient recovery of dynamic behavior in coupled oscillator networks. Phys. Rev. E 88, 032909 (2013).

37. Majdandzic, A. et al. Spontaneous recovery in dynamical networks. Nat. Phys 10, 34-38 (2013)

38. Zou, W., Yao, C. \& Zhan, M. Eliminating delay-induced oscillation death by gradient coupling. Phys. Rev. E 82, 056203 (2010).

39. Zou, W., Senthilkumar, D. V., Zhan, M. \& Kurths, J. Reviving oscillations in coupled nonlinear oscillators. Phys. Rev. Lett. 111, 014101 (2013).

40. Hale, J. K. Functional Differential Equations (Springer, 1971).

41. Atay, F. M. Oscillator death in coupled functional differential equations near Hopf bifurcation. J. Diff. Eqn. 221, 190-209 (2006).

42. Koper, M. Nonlinear phenomena in electrochemical systems. J. Chem. Soc. Faraday Trans. 94, 1369 (1998).

43. Kiss, I. Z., Zhai, Y. \& Hudson, J. L. Emerging coherence in a population of chemical oscillators. Science 296, 1676-1678 (2002).

44. Epstein, I. R. \& Pojman, J. A. Introduction to nonlinear chemical dynamics. Oscillations, Waves, Patterns and Chaos (Oxford Univ. Press, 1998).

45. Atay, F. M. Total and partial amplitude death in networks of diffusively coupled oscillators. Physica. D 183, 1-18 (2003).

46. Daido, H. \& Nakanishi, H. Aging transition and universal scaling in oscillator networks. Phys. Rev. Lett. 93, 104101 (2004).

47. Tanaka, G., Morino, K., Daido, H. \& Aihara, K. Dynamical robustness of coupled heterogeneous oscillators. Phys. Rev. E 89, 052906 (2014).

48. Danino, T., Mondragón-Palomino, O., Tsimring, L. \& Hasty, J. A synchronized quorum of genetic clocks. Nature 463, 326-330 (2010).

49. Mondragón-Palomino, O., Danino, T., Selimkhanov, J., Tsimring, L. \& Hasty, J. Entrainment of a population of synthetic genetic oscillators. Science 333, 1315-1319 (2011).

50. Kopell, N. \& Ermentrout, G. B. Chemical and electrical synapses perform complementary roles in the synchronization of interneuronal networks. Proc. Natl Acad. Sci. USA 101, 15482-15487 (2004).

51. Rosenblum, M. \& Pikovsky, A. Delayed feedback control of collective synchrony: An approach to suppression of pathological brain rhythms. Phys. Rev. E 70, 041904 (2004).

52. Kiss, I. Z., Rusin, C. G., Kori, H. \& Hudson, J. L. Engineering complex dynamical structures: Sequential patterns and desynchronization. Science 316, 1886-1889 (2007). 
53. Pisarchik, A. N. Oscillation death in coupled nonautonomous systems with parametrical modulation. Phys. Lett. A 318, 65-70 (2003).

54. Wei, M. D. \& Lun, J. C. Amplitude death in coupled chaotic solid-state lasers with cavity-configuration-dependent instabilities. Appl. Phys. Lett. 91, 061121 (2007).

55. Bora, M. P. \& Sarmah, D. Oscillation death in a coupled van der Pol-Mathieu system. Pramana 81, 677-690 (2013).

56. Palazzi, M. J. \& Cosenza, M. G. Amplitude death in coupled robust-chaos oscillators. Eur. Phys. J. Special Topics 223, 2831-2836 (2014).

57. Chaudhuri, U. \& Prasad, A. Complicated basins and the phenomenon of amplitude death in coupled hidden attractors. Phys. Lett. A 378, 713-718 (2014).

58. Herrero, R. et al. Experimental observation of the amplitude death effect in two coupled nonlinear oscillators. Phys. Rev. Lett. 84, 5312-5315 (2000).

59. Neufeld, Z., Kiss, I. Z., Zhou, C. S. \& Kurths, J. Synchronization and oscillator death in oscillatory media with stirring. Phys. Rev. Lett. 91, 084101 (2003).

60. Ozden, I. et al. Strong coupling of nonlinear electronic and biological oscillators: Reaching the 'amplitude death' regime. Phys. Rev. Lett. 93, 158102 (2004).

61. Banerjee, T. \& Ghosh, D. Experimental observation of a transition from amplitude to oscillation death in coupled oscillators. Phys. Rev. E 89, 062902 (2014).

62. Hagerstrom, A. M. et al. Experimental observation of chimeras in coupled-map lattices. Nat. Phys. 8, 658-661 (2012).

63. Tinsley, M. R., Nkomo, S. \& Showalter, K. Chimera and phase-cluster states in populations of coupled chemical oscillators. Nat. Phys. 8, 662-665 (2012).

64. Gómez-Gardeñes, J., Gómez, S., Arenas, A. \& Moreno, Y. Explosive synchronization transitions in scale-free networks. Phys. Rev. Lett. 106, 128701 (2011).

65. Leyva, L. et al. Explosive first-order transition to synchrony in networked chaotic oscillators. Phys. Rev. Lett. 108, 168702 (2012).

66. Daido, H. Population dynamics of randomly interacting self-oscillators. Tractable models without frustration. Prog. Theor. Phys. 77, 622-634 (1987).

67. Daido, H. Quasientrainment and slow relaxation in a population of oscillators with random and frustrated interactions. Phys. Rev. Lett. 68, 1073-1076 (1992).

68. Iatsenko, D., McClintock, P. V. E. \& Stefanovska, A. Q. Glassy states and superrelaxation in populations of coupled phase oscillators. Nat. Commun. 5, 4118 (2014)
69. Parker, T. S. \& Chua, L. O. Practical Numerical Algorithms for Chaotic Systems (Springer, 1989).

70. Breda, D. Solution operator approximation for characteristic roots of delay differential equations. Appl. Numer. Math. 56, 305-317 (2006).

\section{Acknowledgements}

We acknowledge financial support from the National Natural Science Foundation of China (No. 11202082, No. 61203235, No. 11371367 and No. 11271290), the Fundamental Research Funds for the Central Universities of China under Grant No. 2014QT005, IRTG1740(DFG-FAPESP), and SERB-DST Fast Track scheme for young scientist under Grant No. ST/FTP/PS-119/2013, NSF CHE-0955555 and Grant No. 229171/2013-3 (CNPq).

\section{Author contributions}

W.Z. conducted theoretical and numerical studies. W.Z. and D.V.S. drafted the manuscript. R.N. and I.Z.K. designed and performed the experiment. Y.T. and A.K. analysed the models and data. J.D. and J.K. directed and supervised the study. All the authors discussed the results, drew conclusions and edited the manuscript.

\section{Additional information}

Supplementary Information accompanies this paper at http://www.nature.com/ naturecommunications

Competing financial interests: The authors declare no competing financial interests.

Reprints and permission information is available online at http://npg.nature.com/ reprintsandpermissions/

How to cite this article: Zou, W. et al. Restoration of rhythmicity in diffusively coupled dynamical networks. Nat. Commun. 6:7709 doi: 10.1038/ncomms8709 (2015).

(i) This work is licensed under a Creative Commons Attribution 4.0 International License. The images or other third party material in this article are included in the article's Creative Commons license, unless indicated otherwise in the credit line; if the material is not included under the Creative Commons license, users will need to obtain permission from the license holder to reproduce the material. To view a copy of this license, visit http://creativecommons.org/licenses/by/4.0/ 